Thermal therapy for breast tumors by using a cylindrical ultrasound phased array with multifocus pattern scanning: a preliminary numerical study

This article has been downloaded from IOPscience. Please scroll down to see the full text article. 2007 Phys. Med. Biol. 524585

(http://iopscience.iop.org/0031-9155/52/15/015)

The Table of Contents and more related content is available

Download details:

IP Address: 140.112.113.225

The article was downloaded on 12/01/2009 at 03:44

Please note that terms and conditions apply. 


\title{
Thermal therapy for breast tumors by using a cylindrical ultrasound phased array with multifocus pattern scanning: a preliminary numerical study
}

\author{
Cheng-Shiao Ho ${ }^{1}$, Kuen-Cheng Ju${ }^{2}$, Tze-Yuan Cheng ${ }^{1}$, Yung-Yaw Chen ${ }^{3}$ \\ and Win-Li Lin ${ }^{1,4}$ \\ ${ }^{1}$ Institute of Biomedical Engineering, National Taiwan University, Taipei, Taiwan \\ 2 Department of Biomedical Engineering, I-Shou University, Kaohsiung, Taiwan \\ ${ }^{3}$ Department of Electrical Engineering, National Taiwan University, Taipei, Taiwan \\ ${ }^{4}$ Division of Medical Engineering Research, National Health Research Institutes, Miaoli, Taiwan \\ E-mail: winli@ntu.edu.tw
}

Received 16 November 2006, in final form 23 April 2007

Published 10 July 2007

Online at stacks.iop.org/PMB/52/4585

\begin{abstract}
The purpose of this study is to investigate the feasibility of using a $1 \mathrm{MHz}$ cylindrical ultrasound phased array with multifocus pattern scanning to produce uniform heating for breast tumor thermal therapy. The breast was submerged in water and surrounded by the cylindrical ultrasound phased array. A multifocus pattern was generated and electrically scanned by the phased array to enlarge the treatment lesion in single heating. To prevent overheating normal tissues, a large planning target volume (PTV) would be divided into several planes with several subunits on each plane and sequentially treated with a cooling phase between two successive heatings of the subunit. Heating results for different target temperatures $\left(T_{\mathrm{tgt}}\right)$, blood perfusion rates and sizes of the PTV have been studied. Furthermore, a superficial breast tumor with different water temperatures was also studied. Results indicated that a higher target temperature would produce a slightly larger thermal lesion, and a higher blood perfusion rate would not affect the heating lesion size but increase the heating time significantly. The acoustic power deposition and temperature elevations in ribs can be minimized by orienting the acoustic beam from the ultrasound phased array approximately parallel to the ribs. In addition, a large acoustic window on the convex-shaped breast surface for the proposed ultrasound phased array and the cooling effect of water would prevent the skin overheating for the production of a lesion at any desired location. This study demonstrated that the proposed cylindrical ultrasound phased array can provide effective heating for breast tumor thermal therapy without overheating the skin and ribs within a reasonable treatment time.
\end{abstract}




\section{Introduction}

Ultrasound thermal therapy which could be a truly non-invasive treatment has emerged as a promising approach for tumor ablation and other medical applications. The main aim of thermal therapy is to raise the temperature in the desired treatment region to result in a critical level of thermal dose that causes tissue coagulation or necrosis. Results of recent studies have shown the potential of the ultrasound thermal therapy for several applications, such as treatments of liver tumor (Kennedy et al 2004, Wu et al 2004), prostate benign hyperplasia (Madersbacher et al 1994, Sanghvi et al 1999), prostate cancer (Vallancien et al 2004), breast cancer (Huber et al 2001, Hynynen et al 2001), kidney cancer (Wu et al 2003, Kohrmann et al 2002), uterine fibroids (Stewart et al 2003, Tempany et al 2003), malignant bone tumor (Wu et al 2001), bladder tumor (Watkin et al 1996) and acoustic hemostasis (Vaezy et al 1997).

Breast is particularly well suited for ultrasound thermal therapy due to the absence of large blood vessels and its convex shape which provides a large window for ultrasound beams. In addition, previous studies of using cylindrical ultrasound transducers for breast hyperthermia have been studied (Ju et al 2006, Lin et al 1998). The preliminary studies of ultrasound thermal therapy for breast tumors using a single spherical transducer (Huber et al 2001) or a hemispherical ultrasound phased array (Malinen et al 2005) have been accomplished. The main limitation of a single spherical transducer is that the focal zone is much smaller, (e.g., the $-3 \mathrm{~dB}$ focus: length $10-15 \mathrm{~mm}$, diameter $2-3 \mathrm{~mm}$ for center frequency $1 \mathrm{MHz}\left(\mathrm{O}^{\prime} \mathrm{Neil}\right.$ 1949)) compared to the tumor size. Therefore, it needs a large number of sonications to achieve the entire treatment, and requires a cooling phase between two successive heatings of the subunit to prevent thermal damage in normal tissues, which results in a long treatment time. A recent clinical report (Zippel and Papa 2005) indicated that it took up to 240 min for treating tumors with a mean size of $22 \mathrm{~mm}$ in diameter. Previous studies have shown that it is feasible by using an ultrasound phased array generating multiple foci simultaneously and electrically switching/scanning the multifocus patterns in the desired heating region for thermal therapy (Daum and Hynynen 1999, Fan and Hynynen 1996, Wan et al 1996). The advantage of this scheme is the capability of generating a relatively larger focal zone within a single sonication, leading to fewer cooling intermissions and reducing the total treatment time significantly.

In addition to a long treatment time, the overheating in bones and the skin burn are also considered as major limitations for ultrasound thermal therapy. The presence of bone in the path of ultrasound beams may induce a significant temperature elevation at the soft-tissue/bone interface, so it attracts a lot of concerns both in therapeutic and diagnostic applications. The acoustic absorption coefficient of bones at frequencies of 0.5 to $5 \mathrm{MHz}$ generally used for ultrasound thermal therapy is one or two order(s) of magnitude greater than that of soft tissues (Goss et al 1978). Moreover, ultrasound beams may reflect at the soft-tissue/bone interface in various degrees depending on their incident angles. Skin burns due to the high absorbed power density in the skin have been reported in some studies (Watkin et al 1997, Gianfelice et al 2003), and may limit the treatment; for instance, by patient pain induced by the regional high temperature. To prevent overheating in bones and skin burns, the acoustic power deposition in these tissues must be minimized.

To effectively abbreviate the total treatment time and avoid overheating in bones and skin burns, a cylindrical ultrasound phased array with a multifocus pattern scanning strategy was proposed for breast tumor thermal therapy. First, the acoustic beams emitted from the cylindrical ultrasound phased array are approximately parallel to the ribs. Second, the convex-shaped breast provides a large window for the acoustic beams emitted from the phased array and hence the average acoustic power deposition on the breast skin could be reduced 


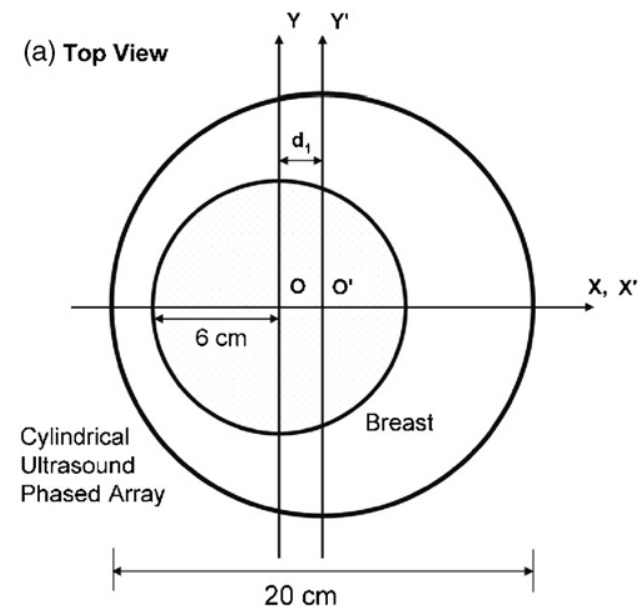

(b) Side View

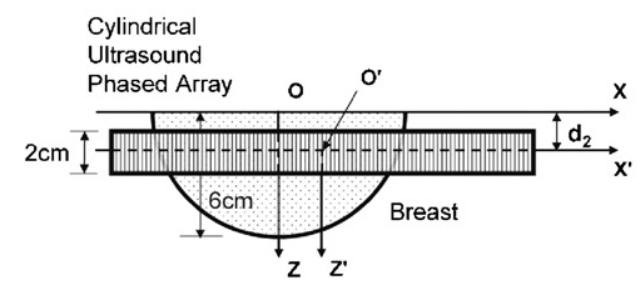

Figure 1. Schematic diagram for the calculation of pressure and temperature fields produced by a cylindrical ultrasound phased array for breast tumor thermal therapy. The breast was modeled as a $12 \mathrm{~cm}$ diameter hemisphere and was surrounded by the phased array. The cylindrical ultrasound phased array was mechanically moved to have its center coincident with the center of the desired heating region before the treatment. $\mathrm{O}-X-Y$ coordinates are fixed on the breast and $\mathrm{O}^{\prime}-X^{\prime}-Y^{\prime}$ coordinates are moved with the cylindrical ultrasound phased array. $\mathrm{O}^{\prime}$ indicates the center of the phased array.

significantly. Furthermore, the coupling water can be used as a cooling medium to produce a great temperature gradient around the skin and effectively prevent skin burn. The simulations were performed to investigate the feasibility of the cylindrical ultrasound phased array for achieving an effective breast tumor thermal therapy within a reasonable treatment time and without overheating ribs and causing skin burn.

\section{Methods}

\subsection{System description}

A schematic diagram of the proposed ultrasound thermal therapy system is illustrated in figure 1. A cylindrical ultrasound phased array consists of 200 elements with a diameter of $20 \mathrm{~cm}$, a height of $2 \mathrm{~cm}$ and a driving frequency of $1 \mathrm{MHz}$. The breast was modeled as a $12 \mathrm{~cm}$ diameter hemisphere and was surrounded by the phased array. Water was filled between the ultrasound phased array and the breast as the coupling medium. The cylindrical ultrasound phased array was mechanically moved to have its geometrical center located at the center of the desired treatment region. Figures 1(a) and (b) show the top view and the side view 
of the ultrasound phased array system moved to the desired treatment region with a shift of $d_{1}$ along the $X$ direction and $d_{2}$ along the $Z$ direction, respectively. With this arrangement, the ultrasound beams emitted from the phased array are approximately parallel to the ribs. Furthermore, the acoustic window on the breast surface was effectively used to reduce the acoustic power intensity on the breast skin. Therefore, the absorbed power density and the temperature elevation of ribs and the skin could be minimized.

\subsection{Acoustic pressure field calculations}

A water-tissue acoustic model was used in this study, and the density and the acoustic speed of the two media are assumed similar. Hence, the reflection and refraction at the watertissue interface could be neglected (Lu et al 1996). Continuous wave sonication was used in the simulation. The acoustic pressure field generated by the ultrasound phased array was calculated by using the Rayleigh-Sommerfeld integral to sum up the contribution of each point source on the surface of the phased array,

$$
p(x, y, z)=\frac{\mathrm{i} \rho c k}{2 \pi} \int_{S} u \frac{\mathrm{e}^{-(\alpha+\mathrm{i} k)\left(\left|r-r^{\prime}\right|\right)}}{\left|r-r^{\prime}\right|} \mathrm{d} S
$$

where $p(x, y, z)$ is the acoustic pressure at point $r=(x, y, z), r^{\prime}$ is the location of the point source $\mathrm{d} S$ on the surface of the phased array, $\rho$ is the density of the medium, $c$ is the acoustic speed of the medium, $k$ is the wave number in the medium $(2 \pi / \lambda$, where $\lambda$ is the wavelength), $u$ is the complex surface velocity of the point source and $\alpha$ is the tissue attenuation coefficient. Equation (1) is difficult to solve in its closed form. A two-dimensional numerical integration over the whole surface of the phased array was used. The surface of the phased array was modeled as an assemblage of point sources with a grid size of $\lambda / 6$ and then all of the waves coming from these point sources to the point $r=(x, y, z)$ are summed. Equation (1) can be expressed as

$$
p(x, y, z)=\mathrm{i} \rho c k \times \frac{\Delta x \Delta y}{2 \pi} \sum_{n=1}^{N} \sum_{m=1}^{M} \frac{u_{n m} \mathrm{e}^{-(\alpha+\mathrm{i} k)\left(\left|r-r^{\prime}\right|\right)}}{\left|r-r^{\prime}\right|}
$$

where $\Delta x \Delta y$ is the area of the point source $n m, u_{n m}$ is the normal velocity of the point source $n m$. Parameters used in the simulation are listed in table 1 (Goss et al 1980, Skinner et al 1998). The absorbed acoustic power deposition $q$ is given by (Nyborg 1981)

$$
q=\frac{\alpha|p|^{2}}{\rho c}
$$

In this study, the scattering effect was not included. Therefore, the acoustic absorption coefficient was assumed to be equal to the attenuation coefficient within the breast. Moreover, the entire breast was also assumed to be homogenous with a constant absorption coefficient. The calculations of acoustic pressure field and absorbed acoustic power deposition were performed with a source grid size of $\lambda / 6$. The driving signals for ultrasound phased array elements generating and scanning a multifocus pattern were synthesized by using a pseudo-inverse method (Ebbini 1989) with a limited maximum output power intensity of $4.9 \mathrm{~W} \mathrm{~cm}^{-2}$ for each element. The maximum acoustic intensity of the multifocus pattern was $225.8 \mathrm{~W} \mathrm{~cm}^{-2}$. 
Table 1. Acoustic and thermal properties of breast tissue used in simulations.

\begin{tabular}{lll}
\hline Symbol & Parameters & Value \\
\hline$c$ & Speed of sound & $1500 \mathrm{~m} \mathrm{~s}^{-1}$ \\
$\rho$ & Tissue density & $1000 \mathrm{~kg} \mathrm{~m}^{-3}$ \\
$\alpha$ & Ultrasound absorption coefficient & $5 \mathrm{~Np} \mathrm{~m}^{-1} \mathrm{MHz}^{-1}$ \\
$k$ & Thermal conductivity & $0.5 \mathrm{~W} \mathrm{~m}^{-1}{ }^{\circ} \mathrm{C}^{-1}$ \\
$c_{\mathrm{t}} / c_{\mathrm{b}}$ & Specific heat (tissue/blood) & $3550 \mathrm{~J} \mathrm{~kg}^{-1}{ }^{\circ} \mathrm{C}^{-1}$ \\
$w_{\mathrm{b}}$ & Blood perfusion rate & $0.5 \mathrm{~kg} \mathrm{~m}^{-3} \mathrm{~s}^{-1}$ \\
\hline
\end{tabular}

\subsection{Calculations of temperature distribution and thermal dose}

The temperature distribution in tissue induced by thermal conduction, blood perfusion and absorbed acoustic power deposition was modeled by using the Pennes bioheat transfer equation (BHTE) (Pennes 1948):

$$
\rho c_{\mathrm{t}} \frac{\partial T}{\partial t}=k \nabla^{2} T-w_{\mathrm{b}} c_{\mathrm{b}}\left(T-T_{\mathrm{ar}}\right)+q,
$$

where $c_{\mathrm{t}}$ and $c_{\mathrm{b}}$ are the specific heat of tissue and blood, respectively, $k$ is the thermal conductivity of the breast tissue; $w_{\mathrm{b}}$ is the blood perfusion rate, and $T_{\mathrm{ar}}$ is the arterial temperature $\left(37^{\circ} \mathrm{C}\right.$ ). Parameters used in simulations are listed in table 1 (Goss et al 1980, Skinner et al 1998). The absorbed acoustic power deposition $q$ obtained from equation (3) was substituted into the BHTE and a three-dimensional finite difference method was used to determine the temperature response. A time step of $50 \mathrm{~ms}$ and a grid spacing of $0.25 \mathrm{~mm}$, $0.25 \mathrm{~mm}$ and $0.5 \mathrm{~mm}$ in $x, y, z$ directions, respectively, were determined to be sufficient for calculation accuracy. The breast tissue properties were assumed to be homogenous without metabolism due to its small contribution to the temperature response. A constant and uniform blood perfusion rate of $0.5 \mathrm{~kg} \mathrm{~m}^{-3} \mathrm{~s}^{-1}$ was assumed in this study; two extreme values (5 and $10 \mathrm{~kg} \mathrm{~m}^{-3} \mathrm{~s}^{-1}$ ) were also evaluated in some cases. The water temperature was set to be $37{ }^{\circ} \mathrm{C}$ on the skin as an energy sink for general cases. For superficial tumor heating, a fixed water temperature of $20^{\circ} \mathrm{C}$ or $37^{\circ} \mathrm{C}$ at $5 \mathrm{~mm}$ away from the breast skin was used to consider the heat conduction from the breast skin to water in order to evaluate the cooling effect on the temperature response. The thermal conductivity of water was $0.55 \mathrm{~W} \mathrm{~m}^{-1}{ }^{\circ} \mathrm{C}^{-1}$ and its specific heat was $3550 \mathrm{~J} \mathrm{~kg}^{-1}{ }^{\circ} \mathrm{C}^{-1}$.

A thermal dose (TD) is used to quantify the effect of temperature and heating duration on tissues and to estimate the necrotic tissue volume, and it was numerically modeled using the following equation (Dewey 1994, Sapareto and Dewey 1984):

$$
\mathrm{TD}=\int_{t_{0}}^{t_{\mathrm{f}}} R^{(T-43)} \mathrm{d} t \approx \sum_{i=0}^{N} R^{T_{t_{i}}-43} \Delta t,
$$

where $t_{0}$ and $t_{\mathrm{f}}$ are the initial and final time, $\Delta t$ is the time step: $50 \mathrm{~ms}, t_{i}=t_{0}+i \Delta t$, $N=\left(t_{\mathrm{f}}-t_{0}\right) / \Delta t, R=2$ for $T_{t_{i}} \geqslant 43{ }^{\circ} \mathrm{C}$, and $R=4$ for $37{ }^{\circ} \mathrm{C}<T_{t_{i}}<43^{\circ} \mathrm{C}$. The range of the thermal dose causing necrosis for soft tissue is from 50 to 240 equivalent minutes at $43^{\circ} \mathrm{C}$ (Damianou and Hynynen 1994, McDannold et al 2002). In this study, the breast tissue was considered to be necrotic when the thermal dose level exceeded 240 equivalent minutes. These simulation codes developed in-house have been previously verified (Liu et al 2004) by comparing with the published paper (Daum and Hynynen 1999). All the simulations were computed using MATLAB ${ }^{\circledR} 7.0$ in a $3 \mathrm{D}$ domain. 

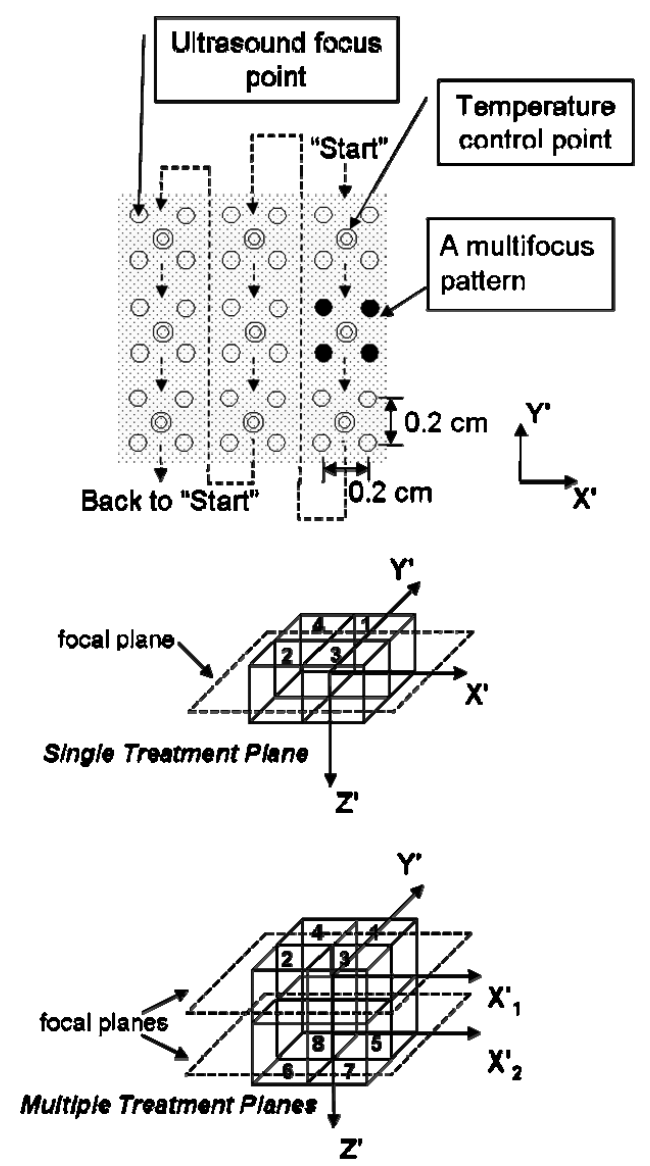

Figure 2. (a) A multifocus pattern formed by four foci during each sonication was electrically scanned to uniformly distribute within the desired heating subunit on the treatment plane. The center of each multifocus pattern was used as a temperature control point (TCP). During the heating phase, the multifocus patterns were sequentially scanned with 1 second-duration sonication at each predetermined location. As the temperature of a TCP reached the target temperature $\left(T_{\text {tgt }}\right)$, the corresponding location would not be sonicated for the following heating. The heating phase stopped when all TCPs had ever reached $T_{\mathrm{tgt}}$. (b) Arrangement for a large PTV treatment. The PTV is divided into several subunits and the number indicates the heating sequence.

\subsection{Heating strategy and treatment planning}

A multifocus pattern was used to enlarge the focal zone for each sonication in this study. Figure 2(a) shows that a multifocus pattern formed by four foci for each sonication was scanned electrically. The spacing between the foci was $2 \mathrm{~mm}$. A previous study showed that this spacing was adequate to provide sufficient thermal dose accumulations between foci (Damianou and Hynynen 1993). The multifocus pattern was uniformly distributed within the desired heating region on the treatment plane. The center of each multifocus pattern was used as a temperature control point (TCP). During the heating phase, the multifocus pattern was sequentially scanned with 1 second-duration sonication at each predetermined location. As the temperature of a TCP reached the target temperature $\left(T_{\mathrm{tgt}}\right)$, the corresponding location would not be sonicated for the following heating. The heating phase stopped when all TCPs 
Table 2. Results of a single heating unit with different target temperatures and blood perfusion rates.

\begin{tabular}{llll}
\hline$w_{\mathrm{b}}\left(\mathrm{kg} \mathrm{m}^{-3} \mathrm{~S}^{-1}\right)$ & $T_{\text {tgt }}\left({ }^{\circ} \mathrm{C}\right)$ & Heating time $(\mathrm{s})$ & Maximum temp $\left({ }^{\circ} \mathrm{C}\right)$ \\
\hline 0.5 & 54 & 65 & 58.2 \\
0.5 & 56 & 77 & 60.5 \\
0.5 & 58 & 87 & 62.7 \\
5 & 56 & 93 & 59.6 \\
10 & 56 & 151 & 58.7 \\
\hline
\end{tabular}

had ever reached $T_{\mathrm{tgt}}$. If the heating region is too large, it may result in overheating of the surrounding normal tissue. A region of $1 \mathrm{~cm} \times 1 \mathrm{~cm}$ on the treatment plane was selected as a desired heating unit. A planning target volume (PTV) larger than this size would be divided into several planes with several subunits on each plane and then heated sequentially. An appropriate cooling phase was needed between two successive heatings of subunits for preventing the undesired temperature build-up outside the PTV. Figure 2(b) illustrates the arrangement of the treatment for a large PTV. The PTV is divided into two treatment planes with eight subunits and the number (from 1 to 8 ) indicates the heating sequence.

\section{Results}

\subsection{Effect of target temperature}

The target temperature $\left(T_{\mathrm{tg}}\right)$ is used as a control parameter to determine the heating sequence and the termination of the heating phase. To evaluate the influence of $T_{\operatorname{tgt}}$ on the treatment for the proposed heating system, a $T_{\text {tgt }}$ of 54,56 or $58^{\circ} \mathrm{C}$ was examined. Figure 3 shows the resulting thermal dose on the $X-Y, X-Z$ and $Y-Z$ planes for a $1 \mathrm{~cm} \times 1 \mathrm{~cm} \times 1 \mathrm{~cm}$ heating unit with its center located at $(0,0,1)$, and a blood perfusion rate of $0.5 \mathrm{~kg} \mathrm{~m}^{-3} \mathrm{~s}^{-1}$. The contour lines from the outermost to the innermost indicate TD $=240 \mathrm{~min}$ for $T_{\text {tgt }}$ equal to 58 , 56 , or $54{ }^{\circ} \mathrm{C}$, respectively. The results indicate that the lesion size (defined as the region with TD greater than $240 \mathrm{~min}$ ) increases with the target temperature in all directions. It is able to produce a $1 \mathrm{~cm} \times 1 \mathrm{~cm}$ lesion on the $X-Y$ plane for $T_{\text {tgt }}$ equal to $56{ }^{\circ} \mathrm{C}$, and the thickness (in the $Z-D$ ) of the lesion is close to $1 \mathrm{~cm}$. Hence a target temperature of $56^{\circ} \mathrm{C}$ was used for the following simulations. The detailed simulation results are listed in table 2.

\subsection{Effect of blood perfusion rate}

The heating strategy of the conventional ultrasound thermal therapy used a single shortduration sonication to generate a small lesion located at the focal zone. Thermal therapy for a large tumor can be achieved by many sonications to form a larger lesion to meet the requirement. Thus, the effect of blood perfusion rates on the thermal lesion formation is not so obvious. In this study, the proposed heating strategy is to scan the multifocus pattern throughout the predetermined heating unit. Generally, it takes about one minute to complete the treatment of a $1 \mathrm{~cm} \times 1 \mathrm{~cm} \times 1 \mathrm{~cm}$ heating unit. Compared to a short-duration sonication (a few seconds), the heating results of a much longer duration sonication might be affected by the blood perfusion rate. To investigate its influence on the thermal lesion formation for the proposed heating system, perfusion rates of $0.5,5$ and $10 \mathrm{~kg} \mathrm{~m}^{-3} \mathrm{~s}^{-1}$ were evaluated. Figures 4(a) and (b) show the thermal dose profiles along the line $Y=0 \mathrm{~cm}$ on the $Z=1 \mathrm{~cm}$ plane, and along the $Z$-axis, respectively. The horizontal dashed line indicates TD $=240 \mathrm{~min}$ and the vertical dashed lines indicate the boundary of a $1 \mathrm{~cm} \times 1 \mathrm{~cm} \times 1 \mathrm{~cm}$ heating unit. 

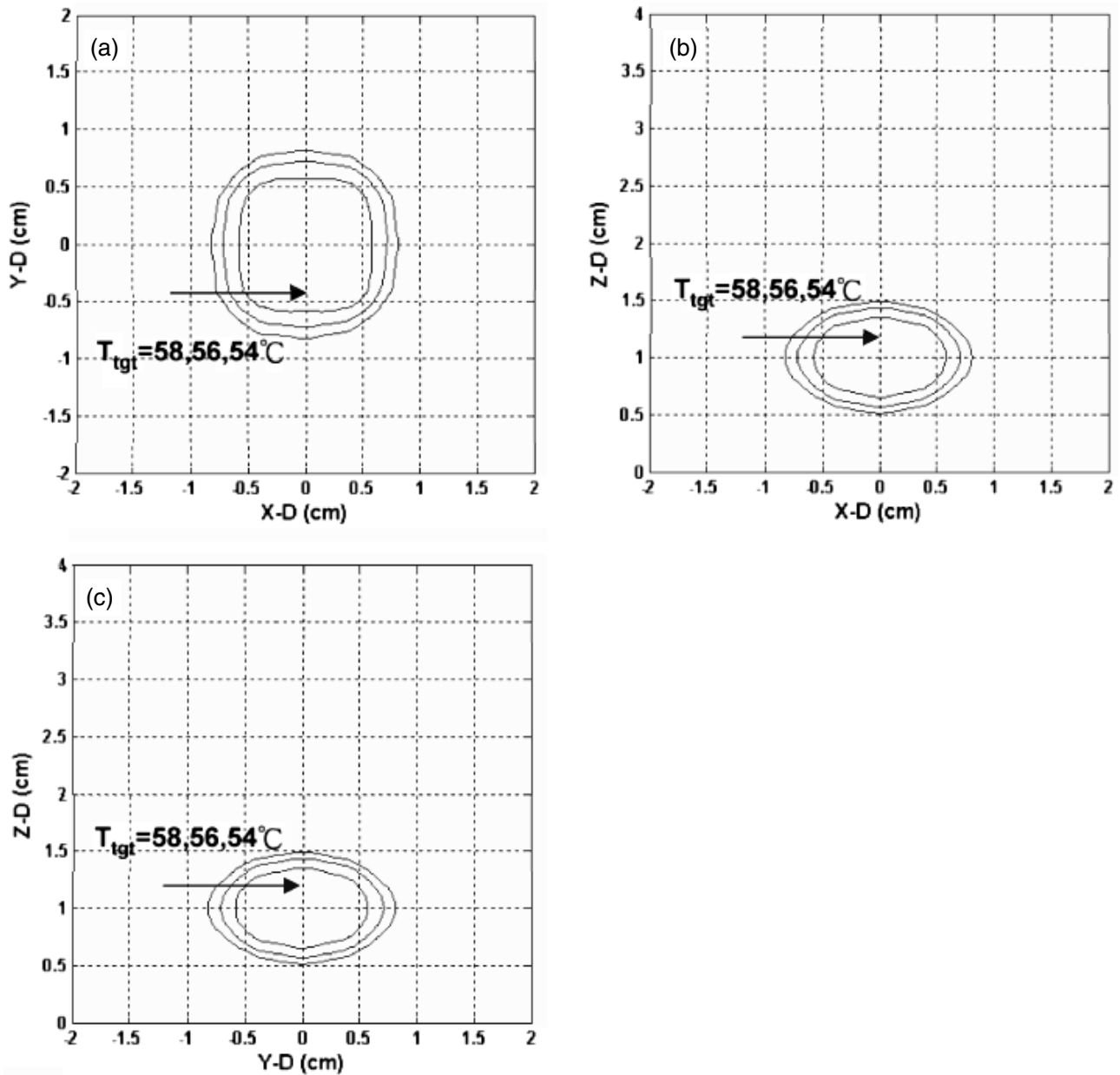

Figure 3. Effect of target temperature $\left(T_{\mathrm{tgt}}\right)$ on the heating results for a single heating unit with its center located at $(0,0,1)$. (a)-(c) show the thermal dose contour of $240 \mathrm{~min}$ for different $T_{\text {tgt }}$ on $Z=1 \mathrm{~cm}, Y=0 \mathrm{~cm}$ and $X=0 \mathrm{~cm}$ planes, respectively. The blood perfusion rate is $0.5 \mathrm{~kg} \mathrm{~m}^{-3} \mathrm{~s}^{-1}$ and $T_{\text {tgt }}$ is set to 58,56 , or $54{ }^{\circ} \mathrm{C}$. The contour lines from the outermost to the innermost indicate $\mathrm{TD}=240 \mathrm{~min}$ for $T_{\mathrm{tgt}}=58,56$, or $54^{\circ} \mathrm{C}$, respectively.

The detailed simulation results were listed in table 2. It was observed that for a lower blood perfusion rate, the maximum temperature during the treatment is higher and it results in a higher maximum thermal dose. However, the thermal dose curves converge together at the boundary of the heating unit for all blood perfusion rates.

\subsection{Treatment for multiple subunits}

To investigate the feasibility of the proposed system for heating a tumor with several subunits on a treatment plane, a PTV of $2 \mathrm{~cm} \times 2 \mathrm{~cm} \times 1 \mathrm{~cm}$ with its center located at $(3,0,1)$ was evaluated. The target temperature is $56{ }^{\circ} \mathrm{C}$, and the blood perfusion rate is $0.5 \mathrm{~kg} \mathrm{~m}^{-3} \mathrm{~s}^{-1}$. The PTV was divided into four subunits with each $1 \mathrm{~cm} \times 1 \mathrm{~cm} \times 1 \mathrm{~cm}$ and sequentially heated as illustrated in figure 2(b). A cooling phase is given between two successive heatings of subunits, and the power is not turned on for the next subunit heating until the peak temperature 

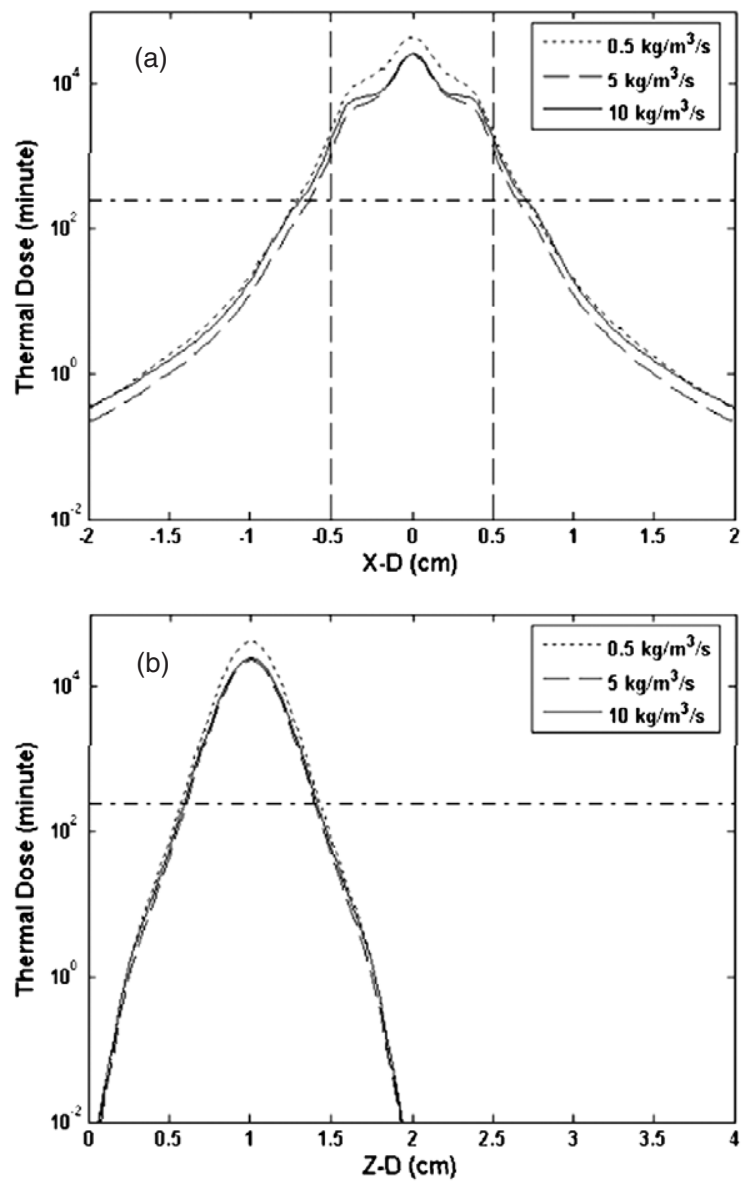

Figure 4. Effect of blood perfusion rate $\left(w_{\mathrm{b}}\right)$ on the heating results for a single heating unit located at $(0,0,1)$ with $T_{\mathrm{tgt}}=56{ }^{\circ} \mathrm{C}$ and $w_{\mathrm{b}}$ equal to $0.5,5$ or $10 \mathrm{~kg} \mathrm{~m}^{-3} \mathrm{~s}^{-1}$. (a) and (b) show the thermal dose profiles along the line $Y=0 \mathrm{~cm}$ on the $Z=1 \mathrm{~cm}$ plane, and along the $Z$-axis, respectively.

Table 3. Results of a single treatment plane with four subunits.

\begin{tabular}{lll}
\hline No of subunits & Heating time (s) & Cooling time (s) \\
\hline$\# 1$ & 75 & 184 \\
$\# 2$ & 62 & 198 \\
$\# 3$ & 57 & 198 \\
$\# 4$ & 61 & 197 \\
\hline
\end{tabular}

of the previously heated subunit is lower than $43{ }^{\circ} \mathrm{C}$. The simulation results on the planes $Z=$ $1 \mathrm{~cm}, Y=0 \mathrm{~cm}$ and $X=3 \mathrm{~cm}$ were shown in figures 5(a)-(c), respectively. The contour lines from the innermost to the outermost indicate TD equal to 240,100 , or $10 \mathrm{~min}$, respectively. The sonication and cooling times required for each subunit treatment are summarized in table 3. It takes about 1 min to complete the heating of a single subunit. Subunit \#1 takes a little longer to complete the heating due to its low initial temperature. The total treatment time from the beginning of sonicating subunit \#1 to the end of sonicating subunit \#4 was 13 min and $55 \mathrm{~s}$. 

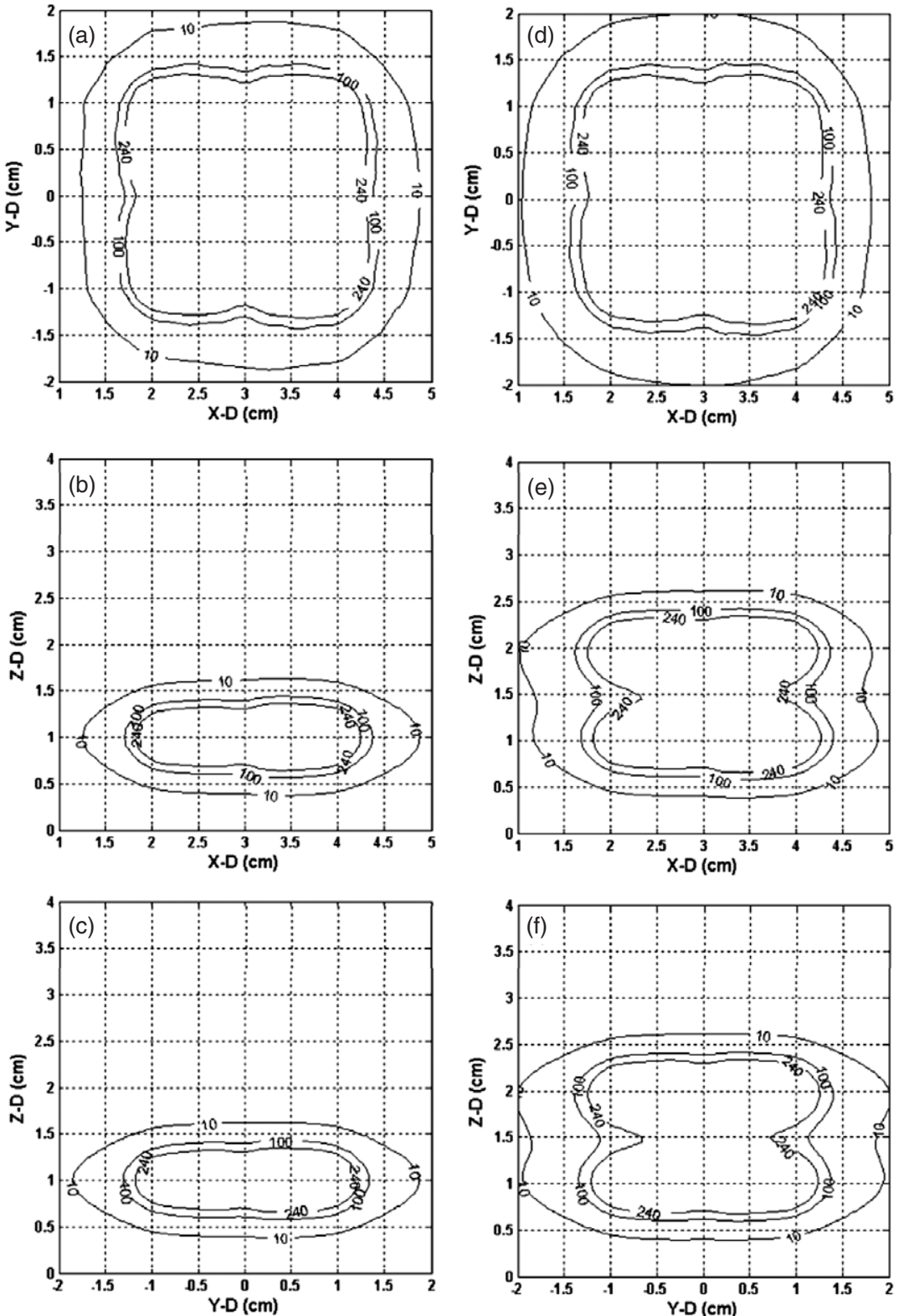

Figure 5. Heating results for large PTVs. (a)-(c) show a desired heating region with four subunits on a plane and its center is located at $(3,0,1)$. (d)-(f) show a desired heating region with four subunits on the treatment plane centered at $(3,0,1)$ and the other four subunits on treatment plane 2 centered at $(3,0,2)$. The target temperature is $56^{\circ} \mathrm{C}$, the blood perfusion rate is $0.5 \mathrm{~kg} \mathrm{~m}^{-3} \mathrm{~s}^{-1}$, and the contour lines indicate TD equal to 240,100 , or $10 \mathrm{~min}$.

To evaluate the heating ability of the proposed ultrasound phased array system for a large tumor, a PTV of $2 \mathrm{~cm} \times 2 \mathrm{~cm} \times 2 \mathrm{~cm}$ with its center located at $(3,0,1.5)$ was studied. The target temperature is $56{ }^{\circ} \mathrm{C}$, and the blood perfusion rate is $0.5 \mathrm{~kg} \mathrm{~m}^{-3} \mathrm{~s}^{-1}$. The PTV was divided into two treatment planes with four subunits on each plane and the heating sequence 
Table 4. Results of the two treatment planes with eight subunits.

\begin{tabular}{llll}
\hline Treatment plane & No of subunits & Heating time $(\mathrm{s})$ & Cooling time (s) \\
\hline 1 & $\# 1$ & 75 & 208 \\
& $\# 2$ & 60 & 240 \\
& $\# 3$ & 56 & 254 \\
& $\# 4$ & 58 & 251 \\
2 & $\# 5$ & 58 & 263 \\
& $\# 6$ & 55 & 249 \\
& $\# 7$ & 54 & 252 \\
& $\# 8$ & 56 & 252 \\
\hline
\end{tabular}

is illustrated in figure 2(b). The heating results on the planes $Z=1 \mathrm{~cm}, Y=0 \mathrm{~cm}$ and $X=$ $3 \mathrm{~cm}$ are shown in figures 5(d)-(f), respectively. The sonication and cooling times required for each subunit treatment are summarized in table 4 . The total treatment time was 36 min and $29 \mathrm{~s}$.

\subsection{Treatment for superficial breast tumor}

Superficial tumors are difficult to be thermally ablated with a spherical ultrasound transducer due to the long ellipsoidal focal zone and a high acoustic absorption coefficient of the skin. To evaluate the heating ability of the proposed ultrasound phased array system for a superficial tumor, a PTV of $1 \mathrm{~cm} \times 1 \mathrm{~cm} \times 1 \mathrm{~cm}$ with its center at $(3.8,0,3.5)$ was studied. The acoustic absorption coefficient for skin was assumed $30 \mathrm{~Np} \mathrm{~m}^{-1} \mathrm{MHz}^{-1}$ with $1 \mathrm{~mm}$ thickness and $5 \mathrm{~Np} \mathrm{~m}^{-1} \mathrm{MHz}^{-1}$ for breast tissue (Goss et al 1978). The shortest distance from the PTV boundary to the skin was $5 \mathrm{~mm}$. In this case, the surrounding water can be used as an energy sink to reduce the temperature elevation in the tissue near water. In this simulation, two water temperatures were evaluated. The heating results on $Z=3.5 \mathrm{~cm}, Y=0 \mathrm{~cm}$ and $X=3.8 \mathrm{~cm}$ planes with water temperatures $37^{\circ} \mathrm{C}$ and $20^{\circ} \mathrm{C}$ are shown in figures $6(\mathrm{a})-(\mathrm{c})$ and figures 6(d)-(f), respectively. The bold solid lines indicate the breast skin. The sonication time is $54 \mathrm{~s}$, the cooling time is $180 \mathrm{~s}$ and the maximum temperature is $61^{\circ} \mathrm{C}$ for the case with water temperature $37{ }^{\circ} \mathrm{C}$; and $58 \mathrm{~s}, 128 \mathrm{~s}$ and $61.4{ }^{\circ} \mathrm{C}$ for water temperature $20^{\circ} \mathrm{C}$. Figure 7 showed the resulting temperature response and thermal dose for different water temperatures on the superficial tumor treatment. Figure 7 (a) is the temperature profile along the $X$ direction at the end of the sonication for the study of figure 6 , and figure 7(b) is the resulting thermal dose profile. The vertical solid lines indicate the skin location $(4.8-4.9 \mathrm{~cm})$, the vertical dashed lines show the desired heating region, and the horizontal line in figure 7(b) is for TD equal to $240 \mathrm{~min}$. The temperature response in the skin at the end of sonication for $20^{\circ} \mathrm{C}$ water was about $6{ }^{\circ} \mathrm{C}$ lower than that for $37^{\circ} \mathrm{C}$ water, and the thermal dose accumulated in the breast skin for $20^{\circ} \mathrm{C}$ water was low enough to be neglected.

\section{Discussion}

The purpose of this study is to investigate the feasibility of the proposed cylindrical ultrasound phased array with a multifocus pattern scanning strategy for breast tumor thermal therapy. The phased array was arranged to embrace the entire breast and to have the desired treatment region located at the geometrical center of the phased array. Hence the acoustic beams of the phased array propagated from all directions into the breast. A multifocus pattern was generated and electrically scanned by the phased array to enlarge the thermal lesion for a single heating. 

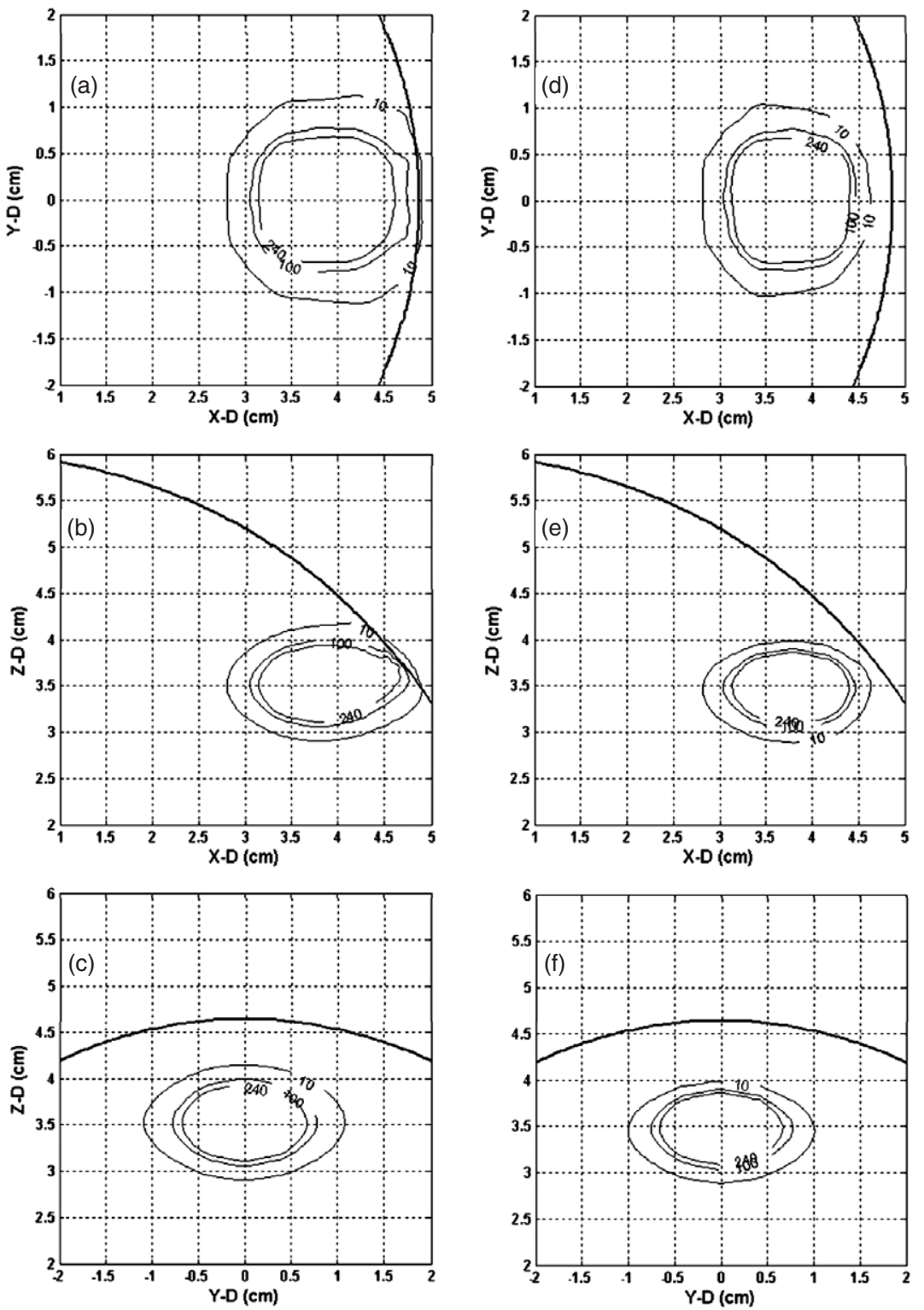

Figure 6. Effect of cooling water temperature on the heating results. (a)-(c) show a single heating unit centered at $(3.8,0,3.5)$ with water temperature $37^{\circ} \mathrm{C}$. (d)-(f) show the same single heating unit with water temperature $20{ }^{\circ} \mathrm{C}$. The blood perfusion rate is $0.5 \mathrm{~kg} \mathrm{~m}^{-3} \mathrm{~s}^{-1}$, and the contour lines indicate TD equal to 240,100 , or $10 \mathrm{~min}$. The bold solid lines indicate the breast skin.

For conformal thermal therapy, the desired treatment region was divided into several subunits and a sequence of heating was used with a cooling phase between two successive heatings. The simulation results shown in figure 5 indicated that the proposed heating system was able to produce appropriately conformal thermal therapy for different sizes and shapes of the treatment region. Tables 3 and 4, showing the sonication and cooling times for each subunit, denote that the entire treatment time is significantly shortened when compared with the results 

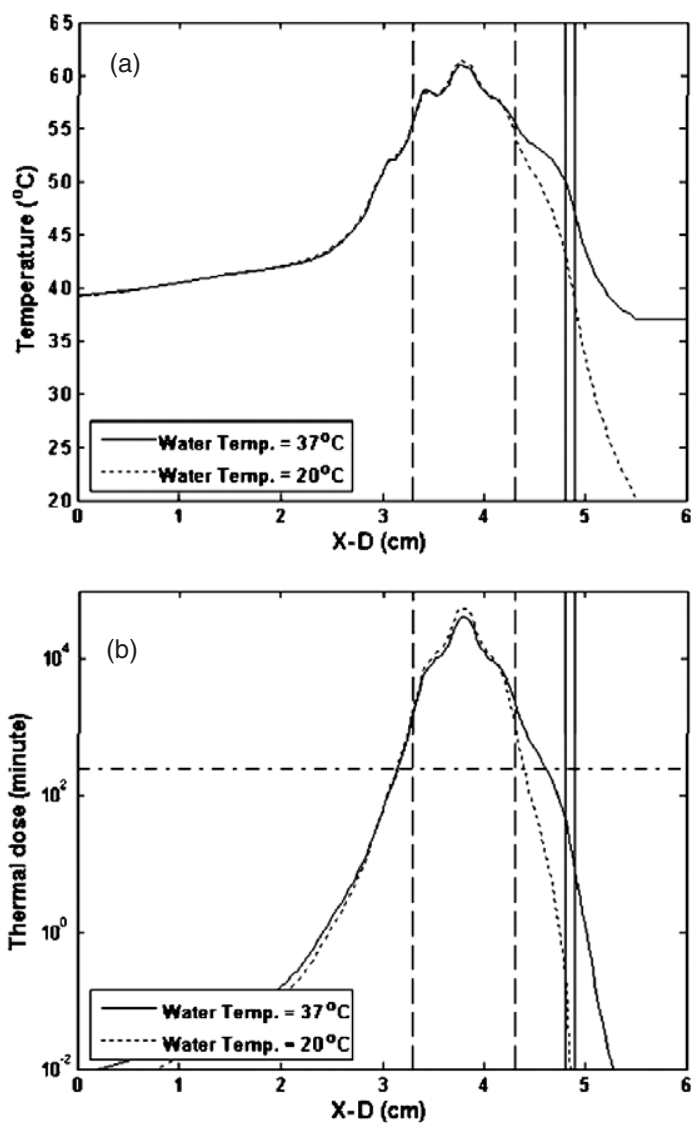

Figure 7. (a) is the temperature profile on the line of $Y=0 \mathrm{~cm}$ and $Z=3.5 \mathrm{~cm}$ at the end of the sonication for the case of figure 6 and (b) is the resulting thermal dose profile. The vertical solid lines indicate the skin location $(4.8-4.9 \mathrm{~cm})$, the vertical dashed lines indicate the desired heating region, and the horizontal line in (b) shows TD equal to $240 \mathrm{~min}$.

of previous studies (Wu et al 2005, Zippel and Papa 2005). The main reasons are that a multifocus pattern scanning strategy was used to generate an enlarged thermal lesion for each single heating and the acoustic beams of the phased array propagated from all directions into the breast. Hence the enlarged thermal lesion for each heating can be rather large, and the total number of cooling phases and the entire cooling time are significantly reduced.

The arrangement between the proposed heating system and the breast provided a maximum window on the breast skin for the acoustic beam emitted from the phased array. This would result in a minimum and uniform power deposition on the skin and the normal tissue surrounding the desired treatment region. Figures 5 and 6 show a sharp drop of thermal dose around the desired treatment region in all directions. Figures 6 and 7 display that the temperature and thermal dose on the skin are not obviously higher than those of the neighboring normal tissue even though the acoustic absorption coefficient for skin is six times that for the breast tissue (30 and $5 \mathrm{~Np} \mathrm{~m}^{-1} \mathrm{MHz}^{-1}$, respectively). This is due to the low power deposition on the skin and the cooling effect of the water. These results indicated that the proposed system and heating strategy is promising to achieve the thermal ablation for the superficial tumor without skin burns and maintain the skin temperature below a critical level to prevent the heating pain. 
The arrangement of the proposed ultrasound phased array also had the acoustic beam emitted from the array approximately parallel to the ribs, and this would result in almost no acoustic power deposition on the ribs. This indicated that there was no overheating problem on the ribs and the breast tissue neighboring the ribs. However, it also denoted that the proposed heating system was not able to heat a tumor which was attached to the ribs. For this situation, two half-cylindrical phased arrays might be used with an appropriate tilt angle and the coupled geometrical center located at the center of the desired treatment region. Further study is required to fully understand the feasibility of treating this special issue by the above arrangement.

\section{Conclusion}

The proposed cylindrical ultrasound phased array has the capability to perform conformal thermal therapy for breast tumors without overheating ribs and causing skin burns within a reasonable treatment time. The phased array with a multifocus pattern scanning strategy can effectively deposit the acoustic power within a suitable heating subunit and reduce the cooling time for the entire treatment. The simulation results showed that a suitable target temperature could be used to determine the resulting lesion size for each subunit heating and the blood perfusion rate had limited effect on the lesion size. The study demonstrated that the proposed ultrasound phased array with a multifocus pattern scanning strategy made remarkable improvement on breast tumor thermal therapy.

\section{Acknowledgments}

This research was supported by the National Science Council grants NSC 93-2213-E-002-069 and NSC 94-2213-E-002-098.

\section{References}

Damianou C and Hynynen K 1993 Focal spacing and near-field heating during pulsed high temperature ultrasound therapy Ultrasound Med. Biol. $19777-87$

Damianou C and Hynynen K 1994 The effect of various physical parameters on the size and shape of necrosed tissue volume during ultrasound surgery $J$. Acoust. Soc. Am. 95 1641-9

Daum D R and Hynynen K 1999 A 256-element ultrasonic phased array system for the treatment of large volumes of deep seated tissue IEEE Trans. Ultrason. Ferroelectr. Freq. Control 46 1254-68

Dewey W C 1994 Arrhenius relationships from the molecule and cell to the clinic Int. J. Hypertherm. 10 457-83

Ebbini E S 1989 Multiple-focus ultrasound-phased-array pattern synthesis_optimal driving-signal distributions for hyperthermia IEEE Trans. Ultrason. Ferroelectr. Freq. Control 36 540-8

Fan X B and Hynynen K 1996 Ultrasound surgery using multiple sonications-treatment time considerations Ultrasound Med. Biol. 22 471-82

Gianfelice D, Khiat A, Amara M, Belblidia A and Boulanger Y 2003 MR imaging-guided focused US ablation of breast cancer: histopathologic assessment of effectiveness-initial experience Radiology 227 849-55

Goss S A, Johnston R L and Dunn F 1978 Comprehensive compilation of empirical ultrasonic properties of mammalian tissues J. Acoust. Soc. Am. 64 423-57

Goss S A, Johnston R L and Dunn F 1980 Compilation of empirical ultrasonic properties of mammalian tissues: II J. Acoust. Soc. Am. 68 93-108

Huber P E, Jenne J W, Rastert R, Simiantonakis I, Sinn H P, Strittmatter H J, von Fournier D, Wannenmacher M F and Debus J 2001 A new noninvasive approach in breast cancer therapy using magnetic resonance imaging-guided focused ultrasound surgery Cancer Res. 61 8441-7

Hynynen K, Pomeroy O, Smith D N, Huber P E, McDannold N J, Kettenbach J, Baum J, Singer S and Jolesz F A 2001 MR imaging-guided focused ultrasound surgery of fibroadenomas in the breast: a feasibility study Radiology $219176-85$ 
Ju K C, Tseng L T, Chen Y Y and Lin W L 2006 Investigation of a scanned cylindrical ultrasound system for breast hyperthermia Phys. Med. Biol. 51 539-55

Kennedy J E, Wu F, ter Haar G R, Gleeson F V, Phillips R R, Middleton M R and Cranston D 2004 High-intensity focused ultrasound for the treatment of liver tumours Ultrasonics 42 931-5

Kohrmann K U, Michel M S, Gaa J, Marlinghaus E and Alken P 2002 High intensity focused ultrasound as noninvasive therapy for multilocal renal cell carcinoma: case study and review of the literature J. Urol. 167 2397-403

Lin W L, Yen J Y, Chen Y Y, Cheng K S and Shieh M J 1998 Specific absorption rate ratio patterns of cylindrical ultrasound transducers for breast tumors Med. Phys. 25 1041-8

Liu H L, Chen Y Y, Yen J Y and Lin W L 2004 Pilot point temperature regulation for thermal lesion control during ultrasound thermal therapy Med. Biol. Eng. Comput. 42 178-88

Lu X Q, Burdette E C, Hansen J L and Svensson G K 1996 Design of an ultrasonic therapy system for breast cancer treatment Int. J. Hypertherm. 12 375-99

Madersbacher S, Kratzik C, Susani M and Marberger M 1994 Tissue ablation in benign prostatic hyperplasia with high intensity focused ultrasound J. Urol. 152 1956-60, discussion 60-1

Malinen M, Huttunen T, Kaipio J P and Hynynen K 2005 Scanning path optimization for ultrasound surgery Phys. Med. Biol. $503473-90$

McDannold N, King R L, Jolesz F A and Hynynen K 2002 The use of quantitative temperature images to predict the optimal power for focused ultrasound surgery: in vivo verification in rabbit muscle and brain $\mathrm{Med}$. Phys. 29 356-65

Nyborg W L 1981 Heat generation by ultrasound in a relaxing medium J. Acoust. Soc. Am. 70 310-2

O’Neil H T 1949 Theory of focusing radiators J. Acoust. Soc. Am. 21 516-26

Pennes H H 1948 Analysis of tissue and arterial blood temperatures in the resting human forearm J. Appl. Physiol. 1 $19-122$

Sanghvi N T, Foster R S, Bihrle R, Casey R, Uchida T, Phillips M H, Syrus J, Zaitsev A V, Marich K W and Fry F J 1999 Noninvasive surgery of prostate tissue by high intensity focused ultrasound: an updated report Eur. J. Ultrasound 9 19-29

Sapareto S A and Dewey W C 1984 Thermal dose determination in cancer therapy Int. J. Radiat. Oncol. Biol. Phys. $10787-800$

Skinner M G, Iizuka M N, Kolios M C and Sherar M D 1998 A theoretical comparison of energy sources-microwave, ultrasound and laser-for interstitial thermal therapy Phys. Med. Biol. 43 3535-47

Stewart E A et al 2003 Focused ultrasound treatment of uterine fibroid tumors: safety and feasibility of a noninvasive thermoablative technique Am. J. Obstet. Gynecol. 189 48-54

Tempany C M C, Stewart E A, McDannold N, Quade B J, Jolesz F A and Hynynen K 2003 MR imaging-guided focused ultrasound surgery of uterine leiomyomas: a feasibility study Radiology 226 897-905

Vaezy S et al 1997 Liver hemostasis using high-intensity focused ultrasound Ultrasound Med. Biol. 23 1413-20

Vallancien G, Prapotnich D, Cathelineau X, Baumert H and Rozet F 2004 Transrectal focused ultrasound combined with transurethral resection of the prostate for the treatment of localized prostate cancer: feasibility study J. Urol. $1712265-7$

Wan H, VanBaren P, Ebbini E S and Cain C A 1996 Ultrasound surgery: comparison of strategies using phased array systems IEEE Trans. Ultrason. Ferroelectr. Freq. Control 43 1085-98

Watkin N A, Morris S B, Rivens I H and ter Haar G R 1997 High-intensity focused ultrasound ablation of the kidney in a large animal model J. Endourol. 11 191-6

Watkin N A, Morris S B, Rivens I H, Woodhouse C R and ter Haar G R 1996 A feasibility study for the non-invasive treatment of superficial bladder tumours with focused ultrasound Br. J. Urol. 78 715-21

Wu F, Chen W Z, Bai J, Zou J Z, Wang Z L, Zhu H and Wang Z B 2001 Pathological changes in human malignant carcinoma treated with high-intensity focused ultrasound Ultrasound Med. Biol. 27 1099-106

Wu F, Wang Z B, Chen W Z, Bai J, Zhu H and Qiao T Y 2003 Preliminary experience using high intensity focused ultrasound for the treatment of patients with advanced stage renal malignancy J. Urol. 170 2237-40

Wu F, Wang Z B, Chen W Z, Zhu H, Bai J, Zou J Z, Li K Q, Jin C B, Xie F L and Su H B 2004 Extracorporeal high intensity focused ultrasound ablation in the treatment of patients with large hepatocellular carcinoma Ann. Surg. Oncol. 11 1061-9

Wu F, Wang Z B, Chen W Z, Zou J Z, Bai J, Zhu H, Li K Q, Jin C B, Xie F L and Su H B 2005 Advanced hepatocellular carcinoma: treatment with high-intensity focused ultrasound ablation combined with transcatheter arterial embolization Radiology 235 659-67

Zippel D B and Papa M Z 2005 The use of MR imaging guided focused ultrasound in breast cancer patients; a preliminary phase one study and review Breast Cancer 12 32-8 